\title{
Modeling term structure dynamics: an infinite dimensional approach *
}

\author{
Rama CONT \\ Centre de Mathématiques Appliquées \\ Ecole Polytechnique \\ F-91128 Palaiseau, France \\ E-mail: Rama.Cont@polytechnique.fr
}

Keywords: stochastic PDE, cylindrical brownian motion, stochastic evolution equations, random fields, term structure of interest rates, forward rates, parabolic equations, random strings, multifactor models.

\footnotetext{
* Part of this work was done while the author was a research fellow at the Institute of Theoretical Physics, Swiss Federal Institute of Technology, Lausanne (EPFL). I am indebted to Nicole El Karoui for introducing me to this subject and sharing with me her experience in interest rate modeling. I also thank Toufik Abboud, Jean-Philippe Bouchaud, Robert Dalang, Stéphane Denise, Olivier Levêque, Carl Mueller, Francesco Russo and Agnès Sulem for useful indications and encouragement. All remaining errors are mine.
} 


\begin{abstract}
We present a family of models for term structure dynamics in an attempt to describe several statistical features observed in empirical studies of forward rate curves by decomposing the deformations of the term structure into the variations of the short rate, the long rate and the fluctuations of the curve around its average shape. This fluctuation is then described as a solution of a stochastic evolution equation in an infinite dimensional space. In the case where deformations are local in maturity, this equation reduces to a stochastic PDE, of which we give the simplest example. We discuss the properties of the solutions and show that they capture in a parsimonious manner the essential features of yield curve dynamics: imperfect correlation between maturities, mean reversion of interest rates and the structure of principal components of term structure deformations. Finally, we discuss calibration issues and show that the model parameters have a natural interpretation in terms of empirically observed quantities.
\end{abstract}

Nous proposons une description de la déformation de la structure par terme des taux d'intérêt en termes d'une équation aux dérivées partielles stochastique. En étudiant en détail le cas linéaire, nous montrons qu'une telle formulation rend compte des observations empiriques sur la dynamique de la courbe des taux forwards avec un nombre restreint de paramètres. Nous discutons en détail le rôle des hypothèses mathématiques et leur impact sur la dynamique des taux d'intérêt. 


\section{Contents}

\begin{tabular}{lll}
\hline & Introduction & 4
\end{tabular}

1.1 Motivations for term structure modeling . . . . . . . . . . . . 4

1.2 Continuous models for discrete observations . . . . . . . . . . 5

1.3 The forward rate curve as an infinite-dimensional process . . . 7

1.4 Relation to previous research . . . . . . . . . . . . . . 8

1.5 Outling . . . . . . . . . . . . . . . . . . . . . . . . . 9

\begin{tabular}{|lll}
2 & Statistical properties of term structure deformations & 10
\end{tabular}

$\begin{array}{lll}3 & \text { Modeling strategy } & 11\end{array}$

3.1 Role of the short rate . . . . . . . . . . . . . . . . . . . . . 11

3.2 Sources of randomness . . . . . . . . . . . . . . . . . . 12

3.3 The Markov property . . . . . . . . . . . . . . . . . . . . . . . 13

3.4 Continuity of term structure deformations . . . . . . . . . . . 14

3.5 Smoothness in maturity . . . . . . . . . . . . . . . . 14

4 A stochastic evolution equation for term structure deforma$\begin{array}{ll}\text { tions } & 15\end{array}$

4.1 Definitions and notations . . . . . . . . . . . . . . 15

$4.2 \quad$ Decomposition of forward rate movements . . . . . . . . . . . 16

4.3 The short rate and the spread: a bivariate Markov process . . 16

4.4 Term structure deformations as Markovian curves . . . . . . . 17

5 Local deformations, stochastic PDEs and string models 20

5.1 Market segmentation and local deformations . . . . . . . . . . 20

5.2 Level, steepness and curvature . . . . . . . . . . . . . 21

5.3 The linear parabolic case . . . . . . . . . . . . . . . . . . . 22

5.4 Eigenmodes and principal components . . . . . . . . . . 23

5.5 Average term structure and mean reversion . . . . . . . . . . . 26

5.6 Random strings: parabolic vs. hyperbolic formulation . . . . . 27

\begin{tabular}{|lll}
\hline 6 & Conclusion and perspectives & 31 \\
\hline
\end{tabular} 


\section{Introduction}

\subsection{Motivations for term structure modeling}

There are two very different motivations for term structure modeling. The first is concerned with the pricing of interest rate derivative securities. In this context, which has been the principal motivation behind term structure models in the mathematical finance literature [CIR, HJM, Vasicek], the main concern has been the development of "coherent" -in the sense of arbitragefree - pricing criteria for securities whose payoffs depend on movements of interest rates.

The second motivation, which could be labeled as "econometric", is the statistical decription of the movements of real interest rates. In contrast to the preceding approach where the emphasis is on cross-sectional coherence of prices given by the model, here the emphasis is on describing and reproducing as closely as possible the time evolution of interest rates from a statistical point of view. Such an approach is useful if one is interested in simulating scenarios, calculating Value-at-Risk of fixed-income positions but also from a theoretical point of view, to gain a better understanding of interest rate fluctations and their relations to other economic variables..

From a mathematical point of view, the first approach corresponds to modeling the dynamics of interest rates under a risk-neutral (or risk-adjusted) measure, while the second approach corresponds to the modeling of real-world term structure dynamics.

Given the complexity of the behavior of the yield curve, there is a conflict between these two criteria which turn out to be difficult to satisfy in the same model. Most of the existing work in the mathematical finance literature on interest rate modeling has adopted the first approach, namely the description of yield curve dynamics under a "risk-adjusted" measure. The reason for this trend is not difficult to understand: contrarily to a stock market, in a bond market the future values of many securities - namely the zero-coupon bonds are known with certainty. The observability of the contemporaneous prices of these bonds then makes it possible to calibrate a model for the risk-adjusted dynamics of interest rates directly to the observed bond prices. However, as correctly pointed out by various authors (see e.g. [Pliska 1997], pp. 201-204), once the risk-adjusted dynamics has been calibrated it is not obvious that

such a model will tell us anything useful about the real-world dynamics of 
interest rates.

In fact, it turns to be difficult to account for a series of empirical facts observed in studies on the term structure of interest rates [Bouchaud et al., 1997] in the framework of standard term structure models of the first type. On one hand, it seems that the constraints implied by the absence of arbitrage in these models are so strong that the latter is obtained at the detriment of a correct representation of the dynamics of the yield curve Bouchaud et al., 1998, El Karoui et al.,1996. On the other hand, some stylized empirical facts about term structure deformations seem to have no theoretical counterpart in classical arbitrage-free models.

\subsection{Continuous models for discrete observations}

Empirical term structure data consist of time series of interest rates of various maturities; for example, in the Eurodollar market interest rates of about 40 different maturities can be obtained on a daily basis. Instead of modeling the data as a sequence of 40-component random vectors, theoretical models tend to represent the interest rate curve as a function of a continuous maturity variable $\theta$ evolving with a continuous time parameter $t$. These models, which offer greater analytical tractability, correspond to the ideal case where fixedincome instruments for continuum of maturities are traded in a continuoustime market. Continuous models for interest rate dynamics can be divided into two classes.

The first class starts by postulating a stochastic model for the short term interest rate: typically, the short rate is considered to follow a stochastic differential equation. The model then specifies a rule for constructing the yield curve from the short rate by an additional set of variables representing the market price(s) of risk. This class includes the Vasicek model Vasicek, the Cox-Ingersoll-Ross model CIR and other variants of these models. Shortrate based models present the advantage of analytical tractability. However, they reduce the dynamics of the whole yield curve to the movement of one it its endpoints, which makes these models rather rigid. A more serious objection to short-rate based models is that empirical studies tend to reject the hypothesis that the short rate is Markovian, let alone a diffusion [Ait-Sahalia 1997.

The second class of models, initiated by Ho \& Lee [Ho \& Lee] and ex-

tended by Heath, Jarrow \& Morton [HJM], take the initial term structure 
and the forward rate volatilities as inputs, model the evolution of forward rates as a family of scalar diffusion process indexed by the maturity date $T$ :

$$
d f_{\mathrm{HJM}}(t, T)=\alpha(t, T) d t+\sigma(t, T) d W_{t}
$$

The forward rate curve is therefore a continuum of diffusion processes driven by a finite number of noise sources; it is therefore intuitively clear that if no constraint is imposed on the drift and diffusion coefficients such a model will present obvious arbitrage opportunities. It was shown in [HJM] that, when the noise source is a finite dimensional Wiener process, then the absence of an arbitrage strategy involving bonds of all maturities imposes the following relation between the drift and the diffusion process of the forward rates:

$$
\alpha(t, T)=\sigma(t, T) \int_{t}^{T} \sigma(t, u) d u+\sigma(t, T) \gamma(t)
$$

where $\gamma(t)$ is some predictable process independent of the time to maturity $\theta=T-t$. Underlying this result are the hypotheses that:

- the number of sources of randomness is finite (in practice, much smaller than the number of maturities)

- arbitrage opportunities involve any number of maturities.

The first hypothesis is a modeling choice which means that for representing the fluctuations of the yield curve, a random vector with around 40 components, we use a model where the number of components $N$ is infinite (in fact a continuum) but the number $k$ of sources of randomness is kept finite. The well-foundedness of this choice is not obvious a priori and justified by observing that Principal Component Analysis (see below) of yield curve movements reveal that around $95 \%$ of the variance of bond yields is explained by 3 factors. As we shall see below, such an observation does not imply that properties other than the covariance structure will be explained by the same small number of factors. Moreover, the relation between drift and volatility (Eq.2) which is fundamental in HJM models is verified if the sources of randomness defining yield curve dynamics are correctly specified. What if one neglects a source of randomness when specifiying the model? This amounts to "projecting" the model on the space spanned by the first

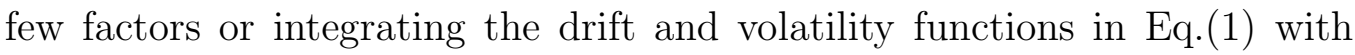
respect to the left-out variable. In this case relation (2) has no reason to hold anymore between the modified drift and volatility. In other words, the 
HJM relation (1) is not robust to misspecification of the number of sources of randomness.

An alternative approach, which we propose here, is to consider the limit where $k$ is also infinite, thus restoring more flexibility to the forward rate curve and at the same time reducing the possibility of easy arbitrage 1 . At the same time, we shall take into account the relative segmentation of the fixed income market into maturity-specific markets to introduce the notion of local deformation and examine its consequences, as explained below.

\subsection{The forward rate curve as an infinite-dimensional process}

In many mathematical models of the yield curve, the term structure of interest rates is often parametrized through the forward rate curve. Let $B(t, T)$ be the price at time $T$ of a (default-free) zero-coupon bond with maturity date $T$. The instantaneous forward rates $f(t, \theta)$ are related to bond prices by

$$
\forall t \in[0, T], B(t, T)=\exp \left(-\int_{0}^{T-t} f(t, u) \quad d u\right)
$$

The forward rate term structure at time $t$ is therefore a function of a continuous variable $\theta$, representing the time to maturity. As such, the forward rate curve $f(t,$.$) naturally lives in an infinite-dimensional space of functions$ and, as remarked by several authors [Duffie \& Kan, Kennedy 1994] there is no reason a priori to believe that its random movements will be restricted to a finite-dimensional subspace or manifold. There are two main reasons why the existing theory has almost exclusively focused on models with a finite number of state variables: the first reason is analytical tractability, the second is that principal component analysis of term structure movements seems to suggest that a few factors are sufficient to explain the covariance structure of interest rates Bouchaud et al., 1997, Litterman \& Scheinkman]. As pointed out above, making a large (formally infinite) number of forward rates dependent on a small number of factors restricts considerable the various configurations of term structure movements, creating a conflict between tractability and a faithful representation of empirical observations.

\footnotetext{
${ }^{1}$ On arbitrage in infinite factor models, see Douady.
} 
Note however that, as pointed out by [Musiela], even with a finite-dimensional noise source in a HJM approach, one cannot ignore the infinite-dimensional character of the forward curve process. This means that, in most such models, the smallest family of forward rates which is Markovian will be an infinite family. However, HJM models endow this infinite family of forward rates with only finitely many degrees of freedom, placing considerable restriction on the type of dynamics it can follow. The difference in the present approach is not only to consider the yield curve as an infinite-dimensional process but also as a process with infinitely many degrees of freedom.

\subsection{Relation to previous research}

Although the intrinsically infinite-dimensional character of continuous term structure models has been remarked by many authors, either it has been often dismissed as being an unrealistic working assumption in yield curve modeling because of the mathematical complexity involved, leading to multifactor models of the term structure (Duffie \& Kan [Duffie \& Kan], El Karoui et al. [El Karoui et al.,1996, El Karoui \& Lacoste,1992, El Karoui et al.,1992]).

Björk et al. Björk et al. proposed a mathematical framework for arbitrage pricing of interest-rate derivatives taking into account the infinitedimensional nature of the yield curve process. However in their representation, following the HJM [HJM] approach, this infinite-dimensional process is driven by a finite-dimensional source of randomness.

An important step was taken by Musiela's Musiela representation of the forward rate as a stochastic process taking its values in a space of functions. Musiela reformulated the HJM equations in terms of a first-order stochastic partial differential equation in the time and maturity variables. Using this same representation, Goldys \& Musiela subsequently derived an infinitedimensional version of the Black-Scholes PDE for a swaption [?].

Another direction which has been developed is the representation of the term structure as a random field with two parameters, time and maturity. First introduced by Kennedy [Kennedy 1994, this approach allows to incorporate an infinite number (or a continuum) of sources of randomness in the evolution of the term structure. Kennedy Kennedy 1994 considers random fields derived from the Brownian sheet, more general random fields are considered by Goldstein Goldstein,1997, who considers as an example random fields which solve a second-order stochastic PDE. Building on these 
examples, Santa Clara \& Sornette Santa Clara \& Sornette, 1998 consider forward rate models in which the forward rate process is driven by a twoparameter noise process ("stochastic string shock"), again pointing out the relation with stochastic PDEs.

Random field models can also be represented as multifactor models with an infinite number of factors. A generalization of the HJM equations to Gaussian infinite factor models was studied by Douady [Douady. Instead of a random field representation, Douady uses an infinite-dimensional representation of the yield curve and introduces a cylindrical Brownian motion as a source of randomness.

An approach which unifies the infinite-dimensional character of the yield curve process and the possibly large number of possible sources of randomess was developed by Bouchaud et al. Bouchaud et al., 1997, Bouchaud et al., 1998 based on an empirical study of deformations of the Eurodollar term structure between 1992 ad 1996. Bouchaud et al. proposed the idea of representing the term structure as a randomly vibrating curve governed by a stochastic partial differential equation containing a second order derivative with respect to maturity and presented some empirical evidence in favor of such models.

Our objective in this paper is to present a model of term structure movements which is both analytically tractable, preserves the infinite-dimensional character of the forward rate curve and reproduces some stylized empirical observations with a small number of parameters. Our approach accounts in a natural manner for the fact that only a small number of factors seem to govern the covariance structure of term structure movements, without imposing any ad hoc unobservable state variable in the model.

More precisely, we will try to demonstrate that specifying the term structure process as a dynamic process with an infinite number of degrees of freedom and eventually proceeding to a finite dimensional approximation afterwards is a more robust modeling procedure than restricting the number of degrees of freedom in the definition of the model, at the risk of misspecifying the factors.

\subsection{Outline}

The paper is structured as follows. In section 2 we recall some important empirical observations about term structure deformations. Based on these observations, we discuss in section 3 what ingredients one should incorpo- 
rate into an interest rate model in order to reproduce the observed statistical properties. We then proceed to give our framework a mathematical formulation in terms of a stochastic evolution equation in a space of smooth functions in section 4. In the case where only local deformations are allowed, this equation reduces to a stochastic partial differential equation: we study a simple example of such an evolution equation in section and show that, albeit its rudimentary structure, it reproduces many properties of term structure deformations in a simple manner.

\section{Statistical properties of term structure de- formations}

As in any applied discipline, empirical observations should be the starting point in the construction of stochastic models in finance. Since our aim here is to model the dynamical behavior of the yield curve, we shall begin by describing some important empirical facts about term structure deformations. The results outlined in this section we mainly refer to [Bouchaud et al., 1997, [Bouchaud et al., 1998 and [Litterman \& Scheinkman].

1. Smoothness in maturity: yield curves do not present highly irregular profiles with respect to maturity. Of course one could argue that with 50 or 60 data points it is difficult to assess the smoothness of a curve; this property should be viewed more as a requirement of market operators. A "jagged" yield curve would be considered as a peculiarity by any market operator. This is reflected in the practice of obtaining implied yield curves by smoothing data points using splines.

2. Irregularity in time: The time evolution of individual forward rates (with a fixed time to maturity) are very irregular. This should be contrasted with the regularity of forward rates with respect to time-tomaturity and reveals an asymmetry between the respective roles of the variables $t$ and $\theta$.

3. Principal components: Principal component analysis of term structure deformations indicates that at least two factors of uncertainty are needed to model term structure deformations. In particular, forward 
rates of different maturities are imperfectly correlated. Empirical studies Bouchaud et al., 1997, Litterman \& Scheinkman] uncover the influence of a level factor which corresponds to parallel shifts of the yield curve, a steepness factor which corresponds to opposite changes in short and long term rates and a curvature factor which influences the curvature of the yield curve. More precisely, the third principal component, when projected on forward rates of different maturities, shows a large component at maturities around one year and small coefficients on the two extremities of the yield curve Bouchaud et al., 1997.

4. Humped term structure of volatility: Forward rates of different maturities are not equally variable. Their variability, as measured for example by the standard deviation of their daily variations, has a humped shape as a function of the maturity, with a maximum at $\theta \simeq 1$ year and decreases with maturity beyond one year [Bouchaud et al., 1997. This hump is always observed to be skewed towards smaller maturities. Moreover, although the observation of a single hump is quite common Moraleda, multiple humps are never observed in the volatility term structure.

5. A multivariate process: while many previous models focused exclusively on the short rate process, trying to represent it as a Markov process, recent econometric studies seem to reject the Markov hypothesis for an interest rate of a given maturity, the short rate in particular, pointing out to the interdependence between interest rates which calls for a multivariate approach.

\section{$3 \quad$ Modeling strategy}

What are the lessons to be drawn from these empirical observations? We will now try to define some criteria which a model should try to respect in order to give a "faithful" statistical representation of interest rate fluctuations.

\subsection{Role of the short rate}

First, the actual dynamics (as opposed to the risk-neutral dynamics) of the forward rate curve cannot be reduced to that of the short rate: the statistical 
evidence points out to the necessity of taking into account more degrees of freedom in order to represent in an adequate fashion the complicated deformations of the term structure. In particular, the imperfect correlation between maturities and the rich variety of term structure deformations shows that a one factor model is too rigid to describe yield curve dynamics.

Furthermore, in practice the value of the short rate is either fixed or at least strongly influenced by an authority exterior to the market (Federal Reserve, central banks), through a mechanism different in nature from that which determines rates of higher maturities which are negotiated on the market. The short rate can therefore be viewed as an exogenous stochastic input which then gives rise to a deformation of the term structure as the market adjusts to its variations. It is therefore plausible from an economic point of view to model separately the dynamics of the short rate.

Second, as shown by Ait Sahalia in a recent study [Ait-Sahalia 1997 the short rate and the long rate (or equivalently, the short rate and the spread) can be reasonably described by a bivariate diffusion such as the one considered by Brennan \& Schwarz 1979] or [Schaefer \& Schwartz].

\subsection{Sources of randomness}

Traditional term structure models such as Vasicek, CIR, HJM define implicitly or explicitly - the random motion of an infinite number of forward rates as diffusions driven by a finite number of independent Brownian motions. This choice may appear surprising since it introduces a lot of constraints on the type of evolution one can ascribe to each point of the forward rate curve and greatly reduces the dimensionality i.e. the number of degrees of freedom of the model, such that the resulting model is not able to reproduce any more the complex dynamics of the term structure. Multifactor models are usually justified by refering to the results of principal component analysis of term structure fluctuations. As remarked above (Sec. 2), it is often observed that the first three principal components explain more than $95 \%$ of the observed variance of forward rates, suggesting that a three factor model would be sufficient. However, one should note that the quantities of interest when dealing with the term structure of interest rates are not the first two moments of the forward rates but typically involve expectations of

\footnotetext{
${ }^{2}$ For a concise review of multifactor term structure models see Duffie \& Kan].
} 
non-linear functions of the forward rate curve: caps and floors are typical examples from this point of view. Hence, although a multifactor model might explain the variance of the forward rate itself, the same model may not be able to explain correctly the variability of portfolio positions involving nonlinear combinations of the same forward rates. In other words, a principal component whose associated eigenvalue is small may have a non-negligible effect on the fluctuations of a non-linear function of forward rates. This question is especially relevant when calculating quantiles and Value-at-Risk measures.

In a multifactor model with $k$ sources of randomness, one can use any $k+1$ instruments to hedge a given risky payoff. However, this is not what traders do in real markets: a given interest-rate contingent payoff is hedged with bonds of the same maturity. ${ }^{3}$ These practices reflect the existence of a risk specific to instruments of a given maturity. The representation of a maturity-specific risk means that, in a continuous-maturity limit one must also allow the number of sources of randomness to grow with the number of maturities; otherwise one loses the localization in maturity of the source of randomness in the model. This point is discussed in more detail in Sec. 5.1.

\subsection{The Markov property}

An important ingredient for the tractability of a model is its Markovian character. Non-Markov processes are difficult to simulate and even harder to manipulate analytically. Of course, any process can be transformed into a Markov process if it is imbedded into a space of sufficiently high dimension; this amounts to injecting a sufficient number of "state variables" into the model. These state variables may or may not be observable quantities; for example one such state variable may be the short rate itself but another one could be an economic variable whose value is not deducible from knowledge of the forward rate curve. If the state variables are not directly observed, they are obtainable in principle from the observed interest rates by a filtering process El Karoui \& Lacoste,1992. Nevertheless the presence of unobserved state variables makes the model more difficult to handle both in terms of interpretation and statistical estimation. This drawback has motivated the development of so-called affine curve models models where one

\footnotetext{
${ }^{3}$ Unless, of course, liquidity considerations impose the trader to do otherwise.
} 
imposes that the state variables be affine functions of the observed yield curve [Duffie \& Kan]. While the affine hypothesis is not necessarily realistic from an empirical point of view, it has the property of directly relating state variables to the observed term structure. We will try to conserve this desirable feature in our model.

\subsection{Continuity of term structure deformations}

Another feature of term structure movements is that, as a curve, the forward rate curve displays a continuous deformation: configurations of the forward rate curve at dates not too far from each other tend to be similar. An animation movie of the successive positions of the forward rate curve displays a continuous movement where the observer can follow a given point on the curvef. This continuity of deformations should be properly defined in mathematical terms and accounted for in a term structure model.

\subsection{Smoothness in maturity}

As already noted above, most applications require the yield curve to have some degree of smoothness e.g. differentiability with respect to the maturity $\theta$. This is not only a purely mathematical requirement but is reflected in market practices of hedging and arbitrage on fixed income instruments. Market practitioners tend to hedge an interest rate risk of a given maturity with instruments of the same maturity or close to it. This important observation means that the maturity is not simply a way of indexing the family of forward rates: market operators expect forward rates whose maturities are close to behave similarly. Moreover, the model should account for the observation that the volatility term structure displays a hump but that multiple humps are never observed. Such an effect could be taken into account by the presence of a term which couples together each forward rate with its neighboring points on the yield curve. We will elaborate more on this aspect in Sec. 5 .

\footnotetext{
${ }^{4}$ Redisplay Fig.4.4 in a postscript version of this article and watch!
} 


\section{A stochastic evolution equation for term structure deformations}

Based on the above considerations, we will now proceed to describe the deformations of the term structure in mathematical form by means of a stochastic evolution equation, translating each of the criteria outlined above into their mathematical equivalents.

\subsection{Definitions and notations}

We will parametrize the evolution of the term structure of interest rates by the instantaneous forward rate curve (FRC), denoted by $f_{t}(\theta)$ where the subscript $t$ denotes time and $\theta \in\left[\theta_{\min }, \theta_{\max }\right]$ the time to maturity. Note that some authors (e.g. [HJM]) specify the forward rate curve as a function of the maturity date $T$; our parametrisation is related to the HJM parametrization in Eq.(11) by

$$
f_{\mathrm{HJM}}(t, T)=f_{t}(\theta=T-t)
$$

As remarked in Musiela, this parametrization has the advantage that the forward rate curve process $f_{t}$ will belong to the same function space (a space of continuous curves defined on $\left.\left[\theta_{\min }, \theta_{\max }\right]\right)$ when $t$ varies, which is not the case of the process $f_{\mathrm{HJM}}(t,$.$) whose domain of definition [t, T]$ shrinks with time. Here $\theta_{\min }$ is the shortest maturity available on the market and $\theta_{\max }$ the longest. $r(t)=f_{t}\left(\theta_{\min }\right)$ will be called the short rate, $l(t)=f_{t}\left(\theta_{\max }\right)$ the long rate. The quantity $s(t)=l(t)-r(t)$ is the spread.

In most interest rate models $\theta_{\min }$ is taken to be 0 and $\theta_{\max }=+\infty$ but this is not necessarily the best choice nor even realistic. First, it is obvious that in empirical applications maturities have a finite span and $\theta_{\max }$ will be typically 30 years or less depending on the applications considered. Second, the finiteness of $\theta_{\max }$ avoids some embarassing mathematical problems related to the $\theta \rightarrow \infty$ limit [Dybvig et al., 1996, El Karoui et al.,1996] which are not necessarily meaningful from an economic point of view. More importantly, we shall see that the $\theta_{\max }=+\infty$ limit is not "innocent" : setting $\theta_{\max }$ to a large but finite value can be qualitatively different from taking it to be infinite. 


\subsection{Decomposition of forward rate movements}

As mentioned above, given the particular nature of the short rate and the well known role of the short rate and the spread as two principal factors, we first proceed to "factor" them out of the model and parametrize the term structure as follows:

$$
f_{t}(\theta)=r(t)+s(t)\left[Y(\theta)+X_{t}(\theta)\right]
$$

where $Y$ is a deterministic shape function defining the average profile of the term structure and $X_{t}(\theta)$ an adapted process decribing the random deviations of the term structure from its long term average shape. With no loss of generality we require:

$$
Y\left(\theta_{\min }\right)=0 \quad Y\left(\theta_{\max }\right)=1
$$

which results in

$$
X_{t}\left(\theta_{\min }\right)=0 \quad X_{t}\left(\theta_{\max }\right)=0
$$

The process $X_{t}(\theta)$ then describes the fluctuations of a random curve with fixed endpoints. We will thus call $X_{t}$ the deformation of the term structure at time $t$. Factoring out the fluctuations of the first two principal components then means modeling separately the process $(r(t), s(t))$ and the deformation process $\left(X_{t}\right)_{t \geq 0}$.

In a Gaussian framework, the uncorrelatedness of the principal components would entail their independence. In particular the first two principal components (which are roughly the spread and the short rate) would be independent from the deformation process $X_{t}$. We will use this assumption as a working hypothesis:

Assumption. The deformation process $X_{t}$ is independent from the short rate $r(t)$ and the spread $s(t)$.

\subsection{The short rate and the spread: a bivariate Markov process}

As in Brennan \& Schwarz 1979", one can consider that the short rate and the long rate (or equivalently, the short rate and the spread, see [Schaefer \& Schwartz) are well described by a bivariate diffusion process: 


$$
\begin{aligned}
& d r_{t}=\mu_{1}\left(r_{t}, s_{t}\right) d t+\sigma_{1,1}\left(r_{t}, s_{t}\right) d W_{t}^{1}+\sigma_{1,2}\left(r_{t}, s_{t}\right) d W_{t}^{2} \\
& d s_{t}=\mu_{2}\left(r_{t}, s_{t}\right) d t+\sigma_{2,1}\left(r_{t}, s_{t}\right) d W_{t}^{1}+\sigma_{2,2}\left(r_{t}, s_{t}\right) d W_{t}^{2}
\end{aligned}
$$

where $W^{1}, W^{2}$ are two independent Wiener processes. This formulation is empirically motivated by the econometric studies refered to above Ait-Sahalia 1997

in which it was shown that the hypothesis of a bivariate diffusion for $\left(r_{t}, s_{t}\right)$ is not rejected by non-parametric tests while it is rejected for $r_{t}$ taken individually.

However, the only hypothesis we need here is the jointly Markovian character of $\left(r_{t}, s_{t}\right)$; ,for example, the noise source in Eq.(8) could be replaced with a non-Gaussian Lévy process without modifying what follows.

\subsection{Term structure deformations as Markovian curves}

We are now left with the deformation process $\left(X_{t}\right)_{t \geq 0}$ to model. The first requirement we impose on $X_{t}$ is its smoothness in maturity: at a given time $t, X_{t}$ is a function defined on $\left[\theta_{\min }, \theta_{\max }\right]$ determined by the forward term structure which, as remarked above, is a "smooth" function of the time to maturity $\theta$. $X_{t}$ should therefore belong to a suitable space $H$ of smooth functions which will then be the state space of our model. In view of interpreting our results in terms of principal component analysis, we would like the state space $H$ to have some Hilbert-like structure in order to define orthogonal projections of $X_{t}$ onto a suitable basis of $H$.

The second requirement we impose is that $X_{t}$ be a Markov process in $H$. This property, as remarked in Musiela, is already verified in the HeathJarrow-Morton [HJM framework for the forward curve process $f_{t}$. Here we require slightly more, namely that the Markovian character respect the factorial decomposition (5). That is, we require the endpoints $(r(t), s(t))$ and the deformation $X_{t}$ to be separately Markovian.

In a functional space, there are of course a wide variety of Markov processes. It is the hypothesis of continuity in time of the deformation process (see (3.4) which enables to single out, the only class among all Markov processes having this property, namely diffusions. More precisely, stating that $X_{t}$ is a $H$-valued diffusion process means that there exist a drift functional $b$ and a volatility functional $\sigma$, defined on $H$, such that the evolution of $X_{t}$ is given by a stochastic differential equation in $H$ (written here in Ito notation): 


$$
d X_{t}=\mu\left(X_{t}\right) d t+\sigma\left(X_{t}\right) d \mathcal{B}_{t}
$$

where $\mathcal{B}_{t}$ is an appropriate generalization of Brownian motion taking values in $H$. Here $\mu$ and $\sigma$ are allowed to depend on the contemporaneous term structure i.e it can be a function of the whole curve $X_{t}(\theta), \theta \in\left[0, \theta^{*}\right]$.

Formally, Eq.(10) is a stochastic differential equation in an (infinitedimensional) functional space $H$. In order to give a proper meaning to Eq.(10), one should start by specifying the nature of the random noise source $\mathcal{B}_{t}$ such that the stochastic integral implicit in Eq.(10) can be properly defined. There are several ways to define a generalization of the Wiener process and a stochastic integral in an infinite dimensional space. The relation between these different constructions was clarified by Yor Yor 1974 who showed that the natural setting for constructing infinite dimensional diffusions is a Hilbert space. Given a (separable) Hilbert space $H$ (for example $H=L^{2}\left(\left[\theta_{\min }, \theta_{\max }\right], \nu\right)$ for some measure $\left.\nu\right)$, one can define a cylindrical Brownian motion on $H$ as a family $\left(\mathcal{B}_{t}\right)_{t \geq 0}$ of random linear functionals $\mathcal{B}_{t}: H \rightarrow R$ satisfying:

1. $\forall \phi \in H, \mathcal{B}_{0}(\phi)=0$

2. $\forall \phi \in H, \mathcal{B}_{t}(\phi)$ is an $\mathcal{F}_{t}$ - adapted scalar stochastic process.

3. $\forall \phi \in H-\{0\}, \frac{\mathcal{B}_{t}(\phi)}{|\phi|}$ is a one-dimensional Brownian motion.

In particular, if one takes any orthonormal basis $\left(e_{n}\right)$ in $H$ then its image $\left(\mathcal{B}_{t}\left(e_{n}\right)\right)_{t \geq 0}$ form a sequence of independent standard Wiener processes in $\mathbf{R}$. This property is useful for building finite-dimensional approximations.

A suitable choice of state space verifying such requirements is a Sobolev space $H^{s}$, namely the space of functions $g \in L^{2}\left(\left[\theta_{\min }, \theta_{\max }\right], \nu\right)$ such that the s-th derivative $g^{(s)}$ is also in $L^{2}\left(\left[\theta_{\min }, \theta_{\max }\right], \nu\right)$, for some measure $\nu$ on $\left[\theta_{\min }, \theta_{\max }\right]$. Given that the derivative $\partial f / \partial \theta$ is assumed to exist in many applications, we would like to require $s \geq 1$. See also the discussion of this point in [Douady], where a similar choice is adopted in a slightly different framework. 


\section{Fluctuations of the term structure}

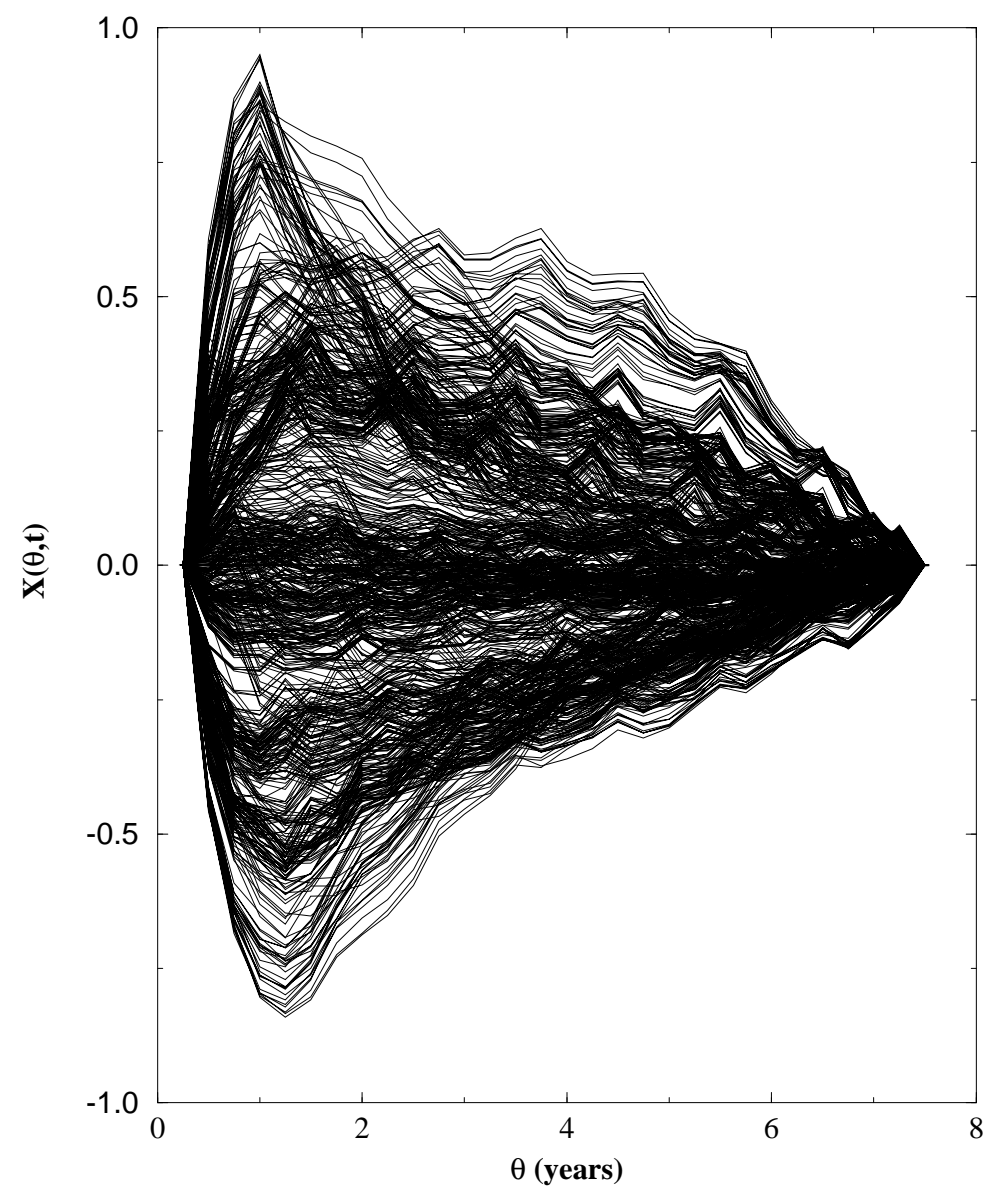

Figure 1: Various configurations adopted by the function $X_{t}(\theta)$ defined in Eq. 5 as obtained from Eurodollar future contracts (1992-7). This figure may be seen as a visualization of the stationary density of the process $X_{t}$. 


\section{Local deformations, stochastic PDEs and string models}

At this level of generality, not much can be said of the properties of the solutions of Eq.(10). In this section we will show how the description of term structure deformations through level, steepness and curvature of the yield curve reduces Eq.(10) to a stochastic partial differential equation, of which some simple examples are given.

\subsection{Market segmentation and local deformations}

As mentioned before, the maturity $\theta$ is not simply a way to index different forward rates and instruments: the fact that fixed income instruments are ordered by maturity is important for market operators. For example, this is reflected in the hedging strategies of operators on the fixed income market: to hedge an interest rate risk of maturity $\theta=8$ months, an operator will tend to use bonds (or other fixed-income instruments) of maturity close to 8 months: 6 months, 9 months. Although this strategy seems quite sensible, it does not correspond to the picture given by multifactor models: in a k-factor model, any $k+1$ instruments can be used to hedge an interest rate contingent claim. For example in a two factor model one could use in principle a 30 year bond, a 10 year bond and a 6 year bond to hedge an instrument with maturity of two years! Needless to say, no sensible trader would follow such a strategy, which shows that in practice the factors which explain $95 \%$ of the variance are not enough to hedge $95 \%$ of the risk of an instrument with a non-linear payoff: this is precisely our principal motivations for introducing maturityspecific sources of randomness i.e. one independent source of randomess per maturity.

The existence of maturity-specific risk naturally leads to a market for such risk. Indeed, some macroeconomic theories of interest rates have considered the interest rate market as being segmented : for example in a first approximation one can consider the market for US Treasury bills, Treasury notes and Treasury bonds as being 3 separate markets where prices are fixed independently. In a continuous-maturity model this would mean that the interest rate market is partitioned into independently evolving markets involving instruments with maturity between $\theta$ and $\theta+d \theta$. 
However this is not strictly true: as shown by principal component analysis, long-term rates react to variations in the short rate in a way that is not explainable simply via parallel shifts and vertical dilations of the term structure. One way to conciliate the interdependence of rates of various maturities with the segmentation of markets across maturities is by considering deformations of the term structure that are local in maturity: a forward rate of maturity $\theta$ is more sensitive to variations of rates with maturity close to $\theta$. We are not dealing here with a strict segmentation of the market into separately evolving markets but a "soft" segmentation which simply implies that the market for each maturity adjusts itself to the variation in rates of maturities immediately above and below it. This means for example that, among all rates of maturity $\geq 1$ year, the 1 year rate will have a higher sensitivity and react more quickly to a variation in the short rate since it is closer in maturity.

\subsection{Level, steepness and curvature}

In mathematical terms, the local deformation hypothesis means that the variation of $X_{t}(\theta)$ will only depend on the behavior of $X_{t}($.$) around \theta$. How can one parametrize the shape of the term structure around a given maturity $\theta$ ? Given that $X_{t}: \theta \rightarrow X_{t}(\theta)$ is assumed to be a smooth curve, its local behavior around $\theta$ can be described by its first few derivatives: $X_{t}(\theta), \partial_{\theta} X_{t}, \partial_{\theta}^{2} X_{t}$,

As noted before, empirical studies seem to identify the level of interest rates, the steepness (slope) of the term structure and its curvature as three significant parameters in the geometry of the yield curve [Litterman \& Scheinkman].

In a market involving instruments of maturity between $\theta$ and $\theta+d \theta$, these three features are described by the level of rates, and the first two derivatives with respect to $\theta$. Combining the local deformation hypothesis formulated in Sec.5.1 with a local description of the term structure by level, steepness and curvature one obtains that the drift and volatility of $X_{t}(\theta)$ can only depend on $X_{t}(\theta), \quad \partial_{\theta} X_{t}, \quad \partial_{\theta}^{2} X_{t}$. Therefore, Eq.(10) becomes a second order stochastic partial differential equation:

$$
\begin{gathered}
d X_{t}=\left[\frac{\partial X_{t}}{\partial \theta}+b\left(X_{t}(\theta), \frac{\partial X_{t}}{\partial \theta}, \frac{\partial^{2} X_{t}}{\partial \theta^{2}}\right)\right] \quad d t+\sigma\left(X_{t}(\theta), \frac{\partial X_{t}}{\partial \theta}, \frac{\partial^{2} X_{t}}{\partial \theta^{2}}\right) d B_{t}(\theta) \\
\forall t \geq 0, X_{t}\left(\theta_{\max }\right)=X_{t}\left(\theta_{\min }\right)=0 \quad X_{t=0}(\theta)=X_{0}(\theta)
\end{gathered}
$$


This equation is the mathematical expression of the fact that deformations are local in maturity and that the deformation at maturity $\theta$ depends on the level, steepness and curvature of the term structure around $\theta$.

In the general case where $b$ and $\sigma$ are smooth but nonlinear functions of their arguments, Eq.(11) is not easy to study: indeed, it is not trivial to define properly what is meant by a solution of Eq.(11) and even less to study their regularity. An approach to the fully non-linear case using the notion of viscosity solution has been recently proposed for the case of a noise-source depending only on $t$ Lions \& Souganidis. In order to point out the differences with HJM-type models resulting from the local deformation hypothesus, we shall consider the case of a forward rate dynamics such as (1) which is perturbated by a term depending on the curvature:

$$
\begin{array}{r}
d X_{t}=\left[\frac{\partial X}{\partial \theta}+b\left(t, \theta, X_{t}(\theta)\right)+\frac{\kappa}{2} \frac{\partial^{2} X}{\partial \theta^{2}}\right] \quad d t+\sigma\left(t, \theta, X_{t}(\theta)\right) d B_{t}(\theta) \\
\forall t \geq 0, X_{t}\left(\theta_{\min }\right)=X_{t}\left(\theta_{\max }\right)=0 \quad X_{t=0}(\theta)=X_{0}(\theta)
\end{array}
$$

Properties of stochastic PDEs such as Eq.(12) have been studied by Pardoux [Pardoux and collaborators. Properties of term structure deformations described by Eq.(12) are studied in Cont 1998. In the following section we study the simplest case where volatility is constant; surprizingly, we will show that this simple case already presents many of the desirable features enumerated in Sec 3 .

\subsection{The linear parabolic case}

In order to illustrate what are the type of dynamics implied by Eq.(12) for term structure deformations, we will now study the simplest example of the above equations which incorporates the influence of local steepness and curvature, namely the case where $\sigma$ is independent of $X_{t}$. For the sake of simplicity we will deal here with the constant volatility case but all the results below remain valid in the case of an arbitrary deterministic function of time $t$ (for details see Cont 1998). The case of constant volatility leads us to the following stochastic partial differential equation ${ }^{5}$ :

$$
\frac{\partial X}{\partial t}=\left[\frac{\partial X}{\partial \theta}+\frac{\kappa}{2} \frac{\partial^{2} X}{\partial \theta^{2}}\right] d t+\sigma_{0} d B_{t}(\theta)
$$

\footnotetext{
${ }^{5} \mathrm{Up}$ to the first derivative in $\theta$, this closely resembles what is known as the stochastic heat equation in the PDE literature.
} 


$$
\begin{aligned}
\forall t \geq 0, & X_{t}\left(\theta_{\text {min }}\right)=X_{t}\left(\theta_{\max }\right)=0 \\
\forall \theta \in\left[\theta_{\text {min }}, \theta_{\text {max }}\right], & X_{t=0}(\theta)=X_{0}(\theta)
\end{aligned}
$$

\subsection{Eigenmodes and principal components}

Let $\theta^{*}=\theta_{\max }-\theta_{\min }$ be the maturity span of the observed forward rate curve. By translating the maturity variable one can assume $\theta_{\min }=0$ without loss of generality in what follows. We consider as state space for our solutions the Hilbert space $H$ of real-valued functions defined on $\left[0, \theta^{*}\right]$ with the scalar product:

$$
<f, g>=\int_{0}^{\theta^{*}} d \theta \quad \exp \left(\frac{2 \theta}{\kappa}\right) f(\theta) g(\theta)
$$

The subscript $H$ in $\langle., .\rangle_{H}$ will be omitted in most of this section. Let $A$ be the operator in $H$ defined by:

$$
A \cdot u=\frac{\partial u}{\partial \theta}+\frac{\kappa}{2} \frac{\partial^{2} u}{\partial \theta^{2}}
$$

It is not difficult to show that $A$ has a discrete spectrum, with eigenvalues and eigenfunctions given by:

$$
\begin{aligned}
A . e_{n} & =-\lambda_{n} e_{n} \\
\lambda_{n} & =\frac{1}{2 \kappa}\left(1+\frac{n^{2} \pi^{2} \kappa^{2}}{\theta^{* 2}}\right) \\
e_{n}(\theta) & =\sqrt{\frac{2}{\theta^{*}}} \sin \left(\frac{n \theta \pi}{\theta^{*}}\right) \exp \left(-\frac{\theta}{\kappa}\right)
\end{aligned}
$$

where $n$ takes all integer values $\geq 1$. Here the eigenfunctions $e_{n}$ have been normalized such that $\left(e_{n}\right)_{n \geq 1}$ is an orthonormal basis of $H$.

$$
<e_{n}, e_{m}>=\delta_{n m}
$$

The functions (eigenmodes) $e_{n}(\theta)$ play the role of the principal components for the deformation process $X_{t}$. That is, if we perform a principal component analysis on a realization of the process $X_{t}$, for a large enough sample the empirical principal components would reproduce the eigenmodes $e_{n}(\theta)$. The 
first two of these eigenmodes are shown in Fig.(5.4). The role of the exponential term in Eq.(20) is clearly visible: the eigenfunctions become "skewed" towards shorter maturities and only a single hump, whose position is determined by the value of $\kappa$, is visible. Recall that this exponential term stems simply from the fact that we are parametrizing the forward rate process by time to maturity $\theta$ instead of maturity date $T$ [Musiela]. In particular, in contrast with multifactor models Moraleda, there is no need to use a complicated volatility structure $\sigma(t, \theta)$ to obtain a volatility hump. The position of the hump gives a (first) simple method for calibrating the value of $\kappa$ to empirical observations.

The Green function (propagator) associated to the operator $A$ may then be expressed in terms of an eigenmode expansion:

$$
G(t, x, y)=\sum_{n \geq 1} \exp \left(-\lambda_{n} t\right) e_{n}(x) e_{n}(y)
$$

and Eq.(13) can be properly defined in the following integral form:

$$
X_{t}(\theta)=\int_{0}^{\theta^{*}} G(t, \theta, y) X_{0}(y) d y+\int_{0}^{t} d s \int_{0}^{\theta^{*}} G(t-s, \theta, y) \sigma_{0} d \mathcal{B}_{s}(y)
$$

Let $X_{t}(\theta)$ be the solution of Eq.(23). Define the coordinates of the solution in the eigenvector basis as:

$$
x_{n}(t)=<X_{t}, e_{n}>=\int_{0}^{\theta^{*}} d \theta \quad X_{t}(\theta) e_{n}(\theta) \quad \exp \left(\frac{2 \theta}{\kappa}\right)
$$

The coefficients $x_{n}(t)$ therefore represents the projection of the deformation process $X_{t}$ on the $n$-th principal component (eigenmode). For each $n, x_{n}(t)$ is then a solution of a linear stochastic differential equation:

$$
d x_{n}(t)=-\lambda_{n} x_{n}(t) d t+\sigma_{0} d W_{t}^{n}
$$

where $W_{t}^{n}=\mathcal{B}_{t}\left(e_{n}\right)$ are independent standard Wiener processes. The $x_{n}(t)$ therefore consitute a sequence of independent Ornstein-Uhlenbeck processes:

$$
x_{n}(t)=e^{-\lambda_{n} t}<e_{n}, X_{0}>+\int_{0}^{t} d s e^{-\lambda_{n}(t-s)} \sigma_{0} d W_{s}^{n}
$$

This last equation has an interesting interpretation. Remember that $x_{n}(t)$ the projection of the deformation process $X_{t}$ on the $n$-th principal component. 
Eq. (26) expresses $x_{n}(t)$ as the sum of two components, the first one being the contribution of the initial term structure to $x_{n}$ and the second one its stationary value. Eq.(26) may then be interpreted by stating that the forward rate curve "forgets" the contribution of the $n$-th principal component to the initial term structure at an exponential rate with characteristic time

$$
\tau_{n}=\frac{1}{\lambda_{n}}=\frac{2 \kappa}{1+\frac{n^{2} \pi^{2} \kappa^{2}}{\theta^{* 2}}}
$$

Therefore, a perturbation of the initial term structure due to the $n$-th principal component will disappear or be smoothed out after a typical time $\tau_{n}$ which decreases with $n$ : "singular" perturbations die out more quickly than smoother ones. This shows the important relation between the smoothing property of the deformation operator $A$ and the decay of its eigenvalues: an operator with a quickly decaying spectrum will guarantee a fast decay (in time) of the singularities appearing in the term structure and restore smoothness in maturity. This gives a second interpretation of the parameter $\kappa$ : in addition to determining the position of the volatility hump, it also determines the decay rate of perturbations of the term structure. This interpretation gives a second, independent method for calibrating the model parameters to empirical data. One can easily imagine more general models where these two roles of $\kappa$ can be attributed to two separately calibrated parameters Cont 1998.

The Ornstein-Uhlenbeck process is the process used to represent the short rate in Vasicek: it possesses the fundamental property of mean reversion which has made it a popular model in interest-rate modeling. In our case, this mean reversion is observed in the principal components of the yield curve: while individual forward rates may have a non-stationary and irregular behavior the yield curve as a whole will converge to a stationary state with a mean-reverting behavior.

Given the explicit form of $\lambda_{n}$, it is easy to show that the series

$$
\begin{aligned}
E\left[X_{t}(\theta)\right] & =\sum_{n \geq 1} E\left[x_{n}(t)\right] e_{n}(\theta) \\
\operatorname{Var}\left[X_{t}(\theta)\right] & =\sum_{n \geq 1} \operatorname{Var}\left[x_{n}(t)\right] e_{n}(\theta)^{2}
\end{aligned}
$$




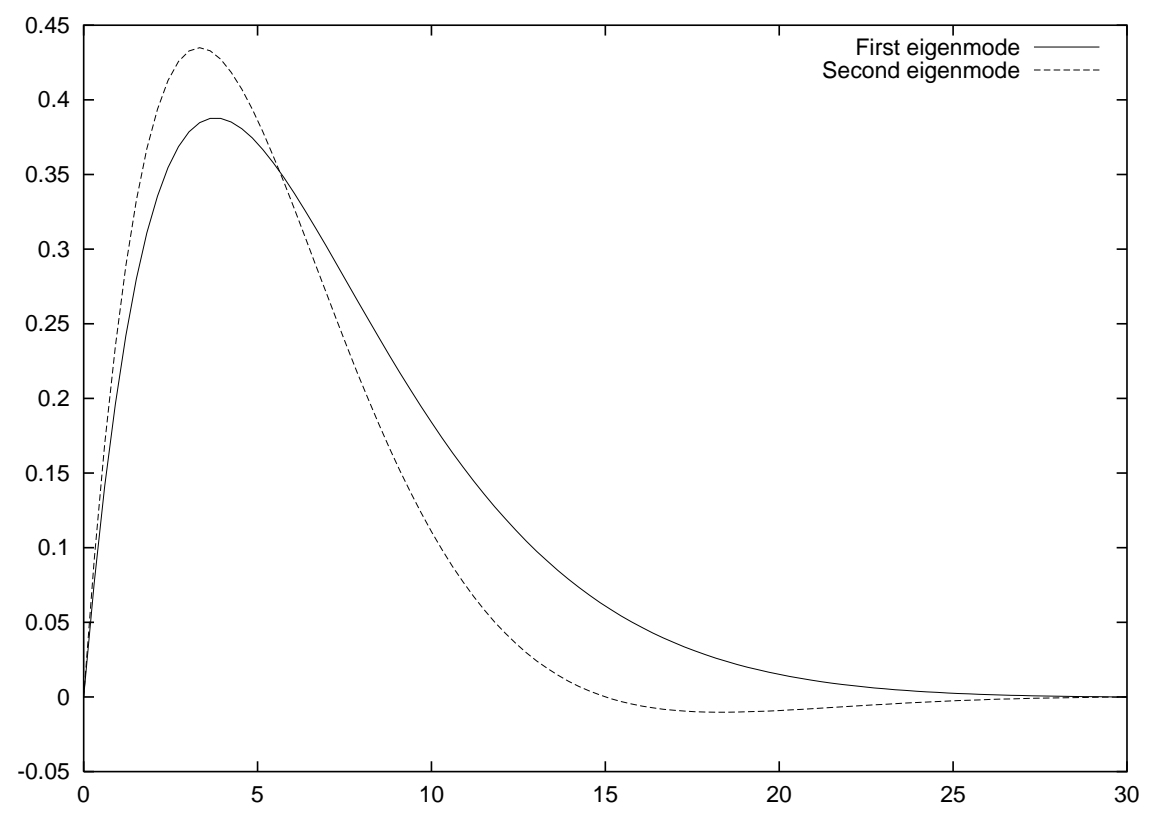

Figure 2: First two eigenmodes of the operator $\mathrm{A}$, with $\mathrm{K}=4$ and $\theta^{*}=30$ years. Note the maxima situated at short maturities.

are absolutely convergent for all $(t, \theta)$ and the sum

$$
X_{t}(\theta)=\sum_{n \geq 1} x_{n}(t) e_{n}(\theta)
$$

defines a unique Gaussian random field with mean and variance given by:

$$
\begin{aligned}
E\left[X_{t}(\theta)\right] & =\sum_{n \geq 1} e^{-\lambda_{n} t}<X_{0}, e_{n}>e_{n}(\theta) \\
\operatorname{Var}\left[X_{t}(\theta)\right] & =\sigma_{0}^{2} \sum_{n \geq 1} \frac{1-\exp \left(-2 \lambda_{n} t\right)}{2 \lambda_{n}} e_{n}(\theta)^{2}
\end{aligned}
$$

\subsection{Average term structure and mean reversion}

Under the above assumptions, one can calculate the average shape of the term structure of forward rates from (Eq. 5).

$$
E[f(t, \theta)]=E[r(t)]+E[s(t)] Y(\theta)
$$


The shape function $Y(\theta)$ can therefore be chosen in order to reproduce the average term structure. In Bouchaud et al., 1997] it was found that the function

$$
Y(\theta)=\sqrt{\frac{\theta}{\theta *}}
$$

gives a good fit of the average shape of the Eurodollar term structure for maturities ranging from 3 months to 10 years. However, the precise analytic form of the shape function $Y$ does not affect the results above. How does the yield curve fluctuate around its average shape? It is easily seen from Eq.(31) that the process $X_{t}$ converges to a Gaussian random field $X_{\infty}$ with mean zero and covariance:

$$
\operatorname{Cov}\left(X_{t}(\theta), X_{t^{\prime}}\left(\theta^{\prime}\right)\right)=\sigma_{0}^{2} \sum_{n \geq 1} \frac{e_{n}(\theta) e_{n}\left(\theta^{\prime}\right) e^{-\lambda_{n}\left(t-t^{\prime}\right)}}{2 \lambda_{n}}
$$

In terms of term structure movements, stationarity of term structure deformations implies a mean-reverting behavior of forward rates. But a model such as the above asserts more: it enables to calculate (therefore calibrate, if one is interested in such quantities) the probability of a given yield curve deformation.

\subsection{Random strings: parabolic vs. hyperbolic formu- lation}

In a recent work [Santa Clara \& Sornette, 1998] it has been proposed to consider stochastic partial differential equations of hyperbolic type to describe the evolution of the forward rate curve. Such an equation differs from the above one through the presence of a second-order time derivative which dominates the dynamics. The model in [Santa Clara \& Sornette, 1998] is formulated in terms of the forward rate process itself (in the spirit of [HJM]) and not in terms of the deformation process $X_{t}$, by examining the effect of inserting a second-order time derivative in the equations of Sec. 5. Let us therefore consider the general case where the evolution equation contains both a propagation term and a diffusion term:

$$
f \frac{\partial^{2} X}{\partial t^{2}}+\frac{\partial X}{\partial t}=\frac{\partial X}{\partial \theta}+\frac{\kappa}{2} \frac{\partial^{2} X}{\partial \theta^{2}}+\sigma_{0} d B_{t}(\theta)
$$




$$
\begin{array}{ll}
\forall t \geq 0, & X_{t}(0)=X_{t}\left(\theta^{*}\right)=0 \\
X_{t=0}(\theta)= & X_{0}(\theta) \frac{\partial X_{t=0}}{\partial t}=Y_{0}
\end{array}
$$

The above equation is analogous to that of a vibrating elastic string, hence the name of "string models" given to such descriptions of term structure movements. The case $f=0$ is the one studied in Sec.5; the case $f \rightarrow \infty$ (the other parameters being appropriately rescaled) is the stochastic wave equation Da Prato \& Zabczyk, the "space" variable being $\theta+t$. Note that the stochastic PDE used in Santa Clara \& Sornette, 1998 is formulated as a PDE perturbated by a two-parameter ("space-time") noise (called a "stochastic string shock") while our Eq.(12) or the general case Eq.(11) was presented above as an evolution equation for a curve in some function space. In the case of a parabolic SPDE, where only the first derivative with respect to time is involved, the two approaches are equivalent for a two-parameter process. Choosing one approach or the other then amounts to viewing the solution of a stochastic PDE either as a random field or as a stochastic process in a function space. Implicit in this choice is whether the object of interest for modeling purposes is an individual interest rate or the deformation of a multivariate object, namely the term structure. The parabolic equation in Sec.5.3 has the merit of emphasizing the asymmetric roles of the variables $\theta$ and $t$, an empirically desirable feature which is not present, as we shall see below, in Santa Clara \& Sornette, 1998.

Manifestly, the operator on the right hand side of Eq.(36) is the same as in Eq.(13). This means that the deformation eigenmodes (the eigenfunctions of $A$ ) will remain the same as in Eq. (13) studied above but the projection of the process $X_{t}$ on each of them will be different. For example, a stationary solution of Eq. (36) will not give the same weight to the eigenmodes as and therefore the results of a Principal Component Analysis of Eq.(36) will differ from that of Eq.(13) in terms of the eigenvalues.

The representation of the equation in terms of its projections on the eigenmode basis $e_{n}$ gives as above a stochastic equation for the scalar process $x_{n}(t)$ :

$$
f \frac{d^{2} y_{n}}{d t^{2}}+\frac{d y_{n}}{d t}=-\lambda_{n} y_{n}(t)+\sigma_{0} \dot{W}_{t}^{n}
$$

This formal second-order stochastic differential equation is interpreted in the 
usual way, as follows. Consider the Green function $U_{n}(t)$ of the operator $f \frac{\partial^{2} u}{\partial t^{2}}+\frac{\partial u}{\partial t}+\lambda_{n} u$, given by:

$$
U_{n}(t)=\frac{1}{f \sqrt{1-4 \lambda_{n} f}}\left[e^{r_{1, n} t}-e^{r_{2, n} t}\right] 1_{t>0}
$$

where $r_{1, n}$ and $r_{2, n}$ being the roots of the associated characteristic equation:

$$
f r^{2}+r+\lambda_{n}=0
$$

The process $x_{n}(t)$ is then given by the stochastic convolution integral:

$$
y_{n}(t)=a_{n} e^{-r_{1, n} t}+b_{n} e^{-r_{2, n} t}+\int U_{n}(t-s) d W_{s}^{n}
$$

which is well-defined since the integrator is square-integrable in $s$. Here $a_{n}$ and $b_{n}$ are defined by the initial term structure. Depending on the values of $f$ and $\kappa$, two scenarios are possible:

1. Oscillating initial conditions: if

$$
f>\frac{\kappa}{2\left(1+\frac{\pi^{2} \kappa^{2}}{\theta^{* 2}}\right)}
$$

then for all $n \geq 1$, Eq. 42 has two complex conjugate roots given by:

$$
\begin{aligned}
& r_{1, n}=\frac{i \sqrt{4 \lambda_{n} f-1}-1}{2 f}=-\frac{1}{2 f}+i \omega_{n} \\
& r_{2, n}=\frac{-i \sqrt{4 \lambda_{n} f-1}-1}{2 f}=-\frac{1}{2 f}-i \omega_{n}
\end{aligned}
$$

The real part gives an exponential damping of the initial conditions which characterizes the mean reverting behavior of $X_{t}$ as in the parabolic case. First remark that, unlike the parabolic case where "bumpy" principal components which contribute the most to non-smoothness in maturity decay more quickly, here all principal components decay with the same speed i.e. a mean reversion time of $2 f$. Recall that $\kappa$ still determines the position of the volatility hump so $\kappa \simeq 1$ year. So (44) implies that $f>6$ months. The mean reversion time of the whole 
curve is thus around a year. However, a new phenomenon appears: the principal components do not simply revert to their mean but oscillate around their mean with a frequency $\omega_{n} / 2 \pi$ which increases with $n$ :

$$
x_{n}(t)=A_{n} e^{-t / 2 f} \cos \left(\omega_{n} t+\phi_{n}\right)
$$

The phase $\phi_{n}$ and amplitude factor $A_{n}$ are determined by (two) initial conditions (see below). The oscillation of the term structure around its mean is not necessarily an undesirable feature of this model and indeed can be justified on economic grounds Cont 1998. But the slow mean reversion combined with increasingly faster oscillations of the higher order principal components leads to non-smoothness in maturity of the solutions of Eq.(36) Da Prato \& Zabczyk : in fact one should expect the cross-sections in time or maturity to have the same irregularity which, as pointed out in Sec.3, is not a desirable feature for a term structure model.

2. Selective damping of principal components: if

$$
f<\frac{\kappa}{2\left(1+\frac{\pi^{2} \kappa^{2}}{\theta^{* 2}}\right)}
$$

then $\exists N>1$ such that for $n \leq N$ Eq.(42) has two real, negative roots whereas for $n>N$ the roots are complex conjugates with negative real parts. The projections of the deformations process $X_{t}$ on the first $N$ eigenmodes will have a mean reverting behavior as in the parabolic case ${ }^{0}$, with a mean reversion time increasing with $n$. For $n>N$, $x_{n}(t)$ will have a damped oscillatory behavior, with a damping time $\tau=2 f$ independent of $n$ and an oscillation frequency increasing with $n$ as above.

Another crucial difference between Eq.(36) and Eq.(12) is the nature of the initial conditions. In the case of the parabolic equation (12) the problem has a well defined solution once the initial term structure is specified through $X_{0}$. This is not sufficient in the case of Eq.(36): one must also specify the derivative with respect to time at $t=0$. In the case of a vibrating string, this

\footnotetext{
${ }^{6}$ In fact the decay of the initial condition is described in this case by the superposition of two decreasing exponentials with time constants given by $r_{1 n}^{-1}$ and $r_{2 n}^{-1}$
} 
means specifying the initial position and the initial velocity of each point of the string. For a model of the forward rate curve, this can be inconvenient: while the initial term structure is the natural input for the initial condition of a dynamic model, the time derivative of the forward rates is not easily evaluated, especially given the irregularity in time of forward rate trajectories which prevents such a model from being calibrated in a numerically stable manner Cont 1998.

We therefore conclude that the question of including a second-order time derivative in Eq.(36) is not simply a matter of taste: the presence of a second derivative radically changes both the dynamic properties of the equation and the nature of the initial conditions needed to calibrate the model, in an empirically undesirable fashion. Our analysis thus pleads for a description of yield curve deformations through a parabolic rather than hyperbolic SPDE.

\section{Conclusion and perspectives}

We have presented a simple stochastic model for describing the fluctuations of the term structure of forward rates: the forward rate curve is described as a random curve oscillating around its long term average. The model studied in Sec. 5 should be viewed as the simplest example of the type of model presented in Sec. 6 . However this simple example has the benefit of emphasizing the role of the second derivative with respect to maturity in the evolution of the term structure: indeed, as we have seen above, it is this second derivative which tames the potentially infinite number of sources of randomness and maintains a regularity in $\theta$ while allowing for independent shocks along maturities. It also gives the correct form for the principal components as well as a qualitatively correct estimate for their associated eigenvalues. These results show the importance of the concept of local deformation explained in Sec.5.1, of which our equation is the simplest example. The model in Sec.5.3 can be easily generalized to the case where the volatility surface $\sigma(t, \theta)$ is an arbitrary deterministic function [Cont 1998]. The introduction of a nonlinear drift $b$ depending on the level of interest rates - as in Eq.(12) - is also possible. More general cases remain to be studied.

As mentioned in the introduction, our objective has been to obtain a faithful continuous-time representation of the statistical properties of the forward rate curve. What remains is to establish the link with the arbitrage pricing 
approach and examine the constraints imposed by absence of arbitrage on models of the type exhibited above. Previous work in this direction Douady indicates that such an analysis requires a careful reconsideration of the class of arbitrage strategies one is willing to consider in the context of a partially segmented market such as the fixed-income market.

\section{References}

[Ait-Sahalia 1997] Ait-Sahalia, Y. (1997) "Do Interest Rates Really Follow Continuous-Time Markov Diffusions?", University of Chicago Working Paper.

[Björk et al.] Björk T., G. Di Masi, Y. Kabanov, W. Runggaldier (1997) "Towards a general theory of bond markets", Finance and Stochastics, 1 (2), 141-174.

[Bouchaud et al., 1997] Bouchaud, J.P., R. Cont, N. El Karoui, M. Potters \& N. Sagna (1997) "Phenomenology of the interest rate curve: a statistical analysis of term structure deformations", Working Paper (http://econwpa.wustl.edu/ewp-fin/9712009).

[Bouchaud et al., 1998] Bouchaud, J.P., R. Cont, N. El Karoui, M. Potters \& N. Sagna (1998) "Strings attached", RISK, July 1998.

[Brennan \& Schwarz 1979] Brennan, M.J. \& E.J. Schwartz (1979) "A continuous-time approach to the pricing of bonds", Journal of Banking and Finance, 3, 133-155.

[Constantinides 1992] Constantinides, G. (1992) "A theory of the nominal structure of interest rates", Review of Financial Studies, 5, 531-552.

[Cont 1998] Cont, R. (1998), "Stochastic PDEs and term structure deformations", Working Paper.

[CIR] Cox, J.C., J.E. Ingersoll \& S.A. Ross (1985) "A Theory of the Term Structure of Interest Rates", Econometrica 53, 385-407.

[Da Prato \& Zabczyk] Da Prato, G. \& Zabczyk, J. (1992) Stochastic equations in infinite dimensions, Cambridge University Press. 
[Douady] Douady, R. (1997) "Yield curve smoothing and residual variance of fixed income positions", Working Paper.

[Duffie \& Kan] Duffie, D. \& R. Kan (1995) "Multifactor models of the term structure" in Howison, Kelly \& Wilmott (Eds.): Mathematical models in finance, London: Chapman \& Hall.

[Dybvig et al., 1996] Dybvig, P., J. Ingersoll and S. Ross (1996) "Long Forward and Zero-Coupon Rates Can Never Fall", Journal of Business 69, $1-26$.

[El Karoui et al.,1996] El Karoui N., Frachot A. \& H. Geman (1996) "A note on the behavior of long zero coupon rates in a no arbitrage framework" CREST Working Paper 96-11.

[El Karoui \& Lacoste,1992] El Karoui, N. \& V. Lacoste (1992) "Multifactor models of the term structure of interest rates", Working paper.

[El Karoui et al.,1992] El Karoui N., R. Myenyi \& R. Viswanathan (1992) "Arbitrage pricing and hedging of interest rate claims with state variables", Working Paper.

[Funaki,1983] Funaki, T. (1983) "Random motions of strings and related stochastic evolution equations", Nagoya Mathematical Journal, 89, 129193.

[Goldstein,1997] Goldstein, R., 1997, "The Term Structure of Interest Rates as a Random Field", Preprint, Ohio State University.

[HJM] Heath, D., R.A. Jarrow and A. Morton (1992) "Bond Pricing and the Term Structure of Interest Rates: A New Methodology for Contingent Claims Valuation", Econometrica 60, 77-105.

[Ho \& Lee] Ho, T.S.Y. \& S. Lee (1986) "Term structure movements and the pricing of interest-rate contingent claims" Journal of Finance, 51, 10111029 .

[Kennedy 1994] Kennedy, D.P. (1994) "The Term Structure of Interest Rates as a Gaussian Random Field", Mathematical Finance 4, 247-258. 
[Lions \& Souganidis] Lions, P.L. \& Souganidis, P.E. (1998) "A viscosity solution approach to fully non-linear stochastic partial differential equations", Cahiers du CEREMADE No.98-22.

[Litterman \& Scheinkman] Litterman R., J. Scheinkman (1991) "Common factors affecting bond returns", Journal of Fixed Income, 1, 49-53.

[Moraleda] Moraleda, J. (1997) Ph.D. thesis, Erasmus University, Rotterdam.

[Musiela] Musiela, M. (1993) "Stochastic PDEs and term structure modeling", Preprint.

[Pardoux] Pardoux, E. (1993) "Stochastic partial differential equations: a review" Bulletin des Sciences Mathématiques, Tome 117, No. 1.

[Peszat \& Zabczyk 1997] Peszat, S. \& Zabczyk, J. (1997) "Stochastic evolution equations with a spatially homogeneous Wiener noise" in Stochastic processes and applications, 72, 187-204.

[Pliska 1997] Pliska, S. (1997) Introduction to mathematical finance, Blackwell Publishers.

[Santa Clara \& Sornette, 1998] Santa Clara, P. \& Sornette, D. (1998) "Stochastic string shocks", UCLA Working Paper.

[Schaefer \& Schwartz] Schaefer, S.M. \& E.S. Schwartz (1987) "Time dependent variance and the pricing of bond options", Journal of Finance, 42, 1113-1128.

[Vasicek] Vasicek, O. (1977) "An Equilibrium Characterization of the Term Structure", Journal of Finance 5, 177-188.

[Walsh] Walsh, J.B. (1986) "An Introduction to Stochastic Partial Differential Equations", in Ecole d'Ete de Probabilité de Saint-Flour, Session XIV, Lecture Notes in Mathematics 1180, Berlin: Springer.

[Yor 1974] Yor, M. (1974) "Existence et unicité de diffusions à valeurs dans un espace de Hilbert", Annales de l'Institut Henri Poincaré, 10, 55-88. 